\title{
Aprendizaje Basado en Problemas (ABP)
}

\section{Estrategia para dinamizar la cátedra universitaria}

\author{
Lic. Aydeé Rivera de Parada ${ }^{1}$ \\ Compilación de experiencias
}

\begin{abstract}
Con esta lógica de trabajo "...no hace falta recurrir a un experto para conocer acerca de un tema, solo hay que saber donde y como buscar esa información" (Pedraz Marcos, A. y otros, 2003). Es el mismo estudiante quien decide sus objetivos de aprendizaje y como hacerlo, conociendo de antemano los principios de las asignaturas que cursa.
\end{abstract}

\section{Introducción}

Según la Red Panamericana para el Aprendizaje Basado en Problemas (PAN-ABP). ${ }^{2}$ El Aprendizaje Basado en Problemas (ABP) es un método dinámico de enseñanza que viene extendiéndose en todo el mundo, tanto en las instituciones de educación superior como en las de educación escolar primaria y secundaria. Este modelo adapta "per se" la investigación como herramienta esencial; los estudiantes construyen sus propios conocimientos mediante la búsqueda de información para el estudio de problemas o necesidades y sus posibles soluciones creando un desafío para ellos. El ABP es una de las mejores formas de integrar la investigación y la docencia en los ámbitos sociales, de manera que promueve una formación de profesionales integrales para desenvolverse en la vida.

Cuando se trabaja con $\mathrm{ABP}$ se invierte el camino convencional del proceso de aprendizaje; mientras tradicionalmente primero se expone la información y posteriormente se busca la aplicación en la resolución de un problema, en el caso del ABP primero se presenta el problema a los estudiantes, éstos identifican sus necesidades de aprendizaje, luego investigan y recopilan la información necesaria para finalmente regresar al problema y darle la solución.

Los problemas que se presentan son problemas reales, complejos, retadores y seleccionados o diseñados por el profesor para el logro de los objetivos de aprendizaje. En el recorrido que viven los alumnos, desde el planteamiento original del problema, hasta su solución, trabajan de manera activa y colaborativa en pequeños grupos, guiados por el profesor o tutor, compartiendo en esa experiencia de aprendizaje la posibilidad de, además de adquirir el conocimiento propio de la materia, practicar y desarrollar habilidades, así como observar y reflexionar sobre actitudes y valores que en el método convencional (expositivo) difícilmente podrían ponerse en acción.

Según experiencias de décadas de aplicación del $\mathrm{ABP}$ en la asignatura de "Legislación y Ética profesional" de la Escuela Universitaria de Enfermería de la Universidad Autónoma de Madrid: esta metodología ha demostrado ser diferente a los métodos tradicionales en los aspectos de currículo, los docentes y los estudiantes "En cuanto al currículo el $\mathrm{ABP}$ aumenta la importancia

1 Licenciada en Ciencias de la Educación. Investigadera, Directora de Investigación UEES

2 La Red Panamericana para el Aprendizaje Basado en Problemas (PAN-ABP) o Pan-American Network or Problem-Based Learning (PAN-PBL) PAN-ABP fue concebida por miembros de la Universidad de Delaware y de la Pontificia Universidad Católica del Perú en el contexto de un proyecto, financiado por Association Liason Office for University Cooperation in Development (ALO), y United States Aid for International Development (USAID), ALO/USAID, para la promoción del uso del ABP en la enseñanza de las matemáticas y de las ciencias en la educación peruana. La red se creó en Lima, Perú, el 9 de diciembre del 2004, durante la primera reunión del Comité Organizador del Congreso Internacional PBL-2006-ABP, contando con la participación de representantes de las universidades anfitrionas de los congresos internacionales de ABP realizados en el continente americano. PAN-ABP tiene como misión promover el Aprendizaje Basado en Problemas en la educación básica [K-12], la educación universitaria (en pre y post grado) y la educación profesional, a lo largo de todo el continente americano. Provee capacitación en ABP y materiales educativos, en español y en inglés, consultoría a instituciones que desean implementar el ABP y colaboración en la organización de Congresos Internacionales cada dos años. Representantes de la UEES asistimos a la videoconferencia titulada Diálogo Global: "El aprendizaje basado en problemas: ¿requiere trasformación curricular o es posible un currículo disciplinar?" 13 de diciembre del 2005 en donde se conocieron experiencias interesantes de ABP de las Universidad de Delaware y de la PUCP entre otras. 
de los objetivos de aprendizaje y la integración del conocimiento, ...Los docentes ven como sus conocimientos no son más una protección, aumenta su vulnerabilidad, la complejidad de la tarea, pero también la satisfacción; por último los estudiantes ven aumentada su responsabilidad, el rango de habilidades necesarias para el aprendizaje, pero también su motivación y satisfacción".

La evaluación en $\mathrm{ABP}$ es un proceso constructivo donde participan los estudiantes de forma individual y grupal, como los docentes, porque la evaluación no debe buscar medir el aprendizaje memorístico sino ser capaz de evaluar en forma constante la relevancia del trabajo y promover la adquisición de destrezas de evaluación crítica, de habilidades clínicas y de mejorar la capacidad de aprendizaje. Los estudiantes trabajando en $\mathrm{ABP}$ tienen, además, la posibilidad de comprender la importancia de trabajar colaborativamente y de comprometerse con sus propios procesos de aprendizaje.

Entre quienes lo promueven, existe un profundo debate respecto a la posibilidad o no de implementar este método al interior de un currículo organizado disciplinalmente. Hay quienes sostienen que ello no es posible, que desvirtúa el método mismo y que la única opción para su implementación es la transformación previa del currículo en una perspectiva interdisciplinaria.

La evaluación en $A B P$ es un proceso constructivo donde participan los estudiantes de forma individual $y$ grupal, como los docentes, porque la evaluación no debe buscar medir el aprendizaje memorístico sino ser capaz de evaluar en forma constante la relevancia del trabajo...

La Universidad de Delaware (EEUU), la Universidad de Colima (México) y la Pontificia Universidad Católica de Perú (PUCP) tienen experiencias muy diversas en la implementación del Aprendizaje Basado en Problemas. Mientras que en la Universidad de Delaware, desde 1992, se ha venido extendiendo como método de enseñanza en las asignaturas de currículos disiciplinares; en la Universidad de Colima supuso la transformación previa de los currículos de Ciencias de la Salud (Medicina, Enfermería y
Psicología); y en la PUCP se inició en 2001 su implementación en asignaturas de currículos disciplinares, pero con una tensión muy fuerte en la dirección de transformar el currículo hacia un currículo interdisciplinario.

\section{Caracteristicas del aprendizaje basado en problemas $^{3}$}

- Mezcla el carácter personal del aprendizaje con el colectivo;

- Posee un importante papel catalizador de los conocimientos o representaciones previas;

- Valora la importancia de recursos funcionales a disposición;

- Asigna un rol preponderante al contexto de los estudiantes y a la oportunidad de la experiencia concreta;

- Permite el desarrollo de competencias complejas;

- Exige la aplicación de pasos lógicos de la investigación;

- Valora el carácter interactivo y el trabajo colaborativo;

- Permite establecer relaciones de significado entre el interés personal, de estudios y de aplicación;

- Valora la importancia de un resultado, y

- Permite la reflexión sobre lo que se está aprendiendo mediante la solución del problema.

\section{A continuación se presentan Algunas experiencias de $A B P$}

A) Análisis de una propuesta de trabajo partir de un programa de análisis de necesidades.

Limón Domínguez (1999), diseñó criterios de selección de contenidos curriculares a partir de un programa de análisis de necesidades para la cátedra de Educación Ambiental, en la Universidad de Sevilla, y compartió la experiencia en el XVIII Seminario interuniversitario de Teoría de la Educación, titulado: "La educación obligatoria. Competencias Básicas del Alumno" en la Universidad de Extremadura en 1999, en donde se presentaron experiencias de docentes y alumnos.

Ante la posibilidad de un trabajo de selección de contenidos se consideró necesario partir de un análisis de necesidades donde se refleje tanto las opiniones del profesor como de los estudiantes. Atendiendo sobre todo a un modelo de "enseñanza para la comprensión"; está la necesidad de partir de las ideas previas de los estudiantes y de su capacidad de análisis. Según Limón Domínguez, esta línea la defienden, entre otros, autores como Pérez Gómez, A.I. (1985), Popkewitz, Th.S. (1988), y Apple, M. (1987). Por lo tanto, a la hora de fortalecer un nuevo

3 Educarchile es un portal autónomo, pluralista y de servicio público que cuenta con la colaboración de los sectores público, privado y filantrópico. Concurren a su creación el Ministerio de Educación de Chile y la Fundación Chile. Nace de la confluencia de los sitios educativos de la Red Enlaces del Ministerio de Educación y del Programa de Educación de la Fundación Chile. http://www.educarchile.cl/home/escritorio_docente/ Visitado el 09/06/2006. 
curriculum (sobre todo, tratando de interaccionar a partir de realidades concretas), ha de alejarse del análisis de un "modelo de enseñanza centrado en el profesor y dentro de una estrategia expositiva"; de no hacerlo así, nos encaminaríamos hacia un "modelo de interacción condicionado por la enseñanza verbal". Este desvío supondría el alejamiento de un compromiso real en la valoración crítica de la realidad y la búsqueda de estrategias de desarrollo eficaces, ya sean cognitivas, afectivas o procedimentales.

¿Porqué partir de la evaluación de necesidades para la selección de contenidos?

Por necesidad, entendemos todo aquello que se demanda desde una perspectiva justa y socialmente considerada. En el "Diccionario de Ética" (1994), citado por Limón, se dice que "el obrar humano está determinado por las necesidades", y que "el ser humano tiene, mediante el pensamiento y el lenguaje, la posibilidad de iniciar éstas como motivo de sus acciones, de realizarlas, modificarlas o contenerlas". Así, se entiende la interrogación por las necesidades, desde el punto de vista de que el obrar moral debería comprender y realizar las necesidades verdaderas y racionales de los hombres.

Los resultados de la propuesta se analizaron mediante relatos, entre los que se seleccionaron una serie de informantes clave, que representaban, los criterios mayoritarios. Según docentes el objetivo era llevar esa información complementaria a la clase y trabajarla en forma de debate para que, a raíz de lo dicho, cada uno sacara sus propias conclusiones. Según experiencia de alumnos: "Se trataba de lanzar propuestas y alternativas a nuestro alcance para indagar y trabajar detalles y aspectos más concretos dentro de la globalización, con distintas fuentes de información (lecturas, experiencias personales, etc.); el clima de trabajo giraba en torno a "una construcción del aprendizaje y de nuestra propia visión, nuestra postura ante la asignatura, pero a su vez, de trasladarlo a todos los aspectos de la vida", así lo expresaba uno de los estudiantes, en uno de los relatos recogidos.

En relación con la experiencia anterior, se considera la delimitación de estrategias que permitan implantar un proceso de formación en la acción desde una propuesta concreta de contenidos dentro de la asignatura, que ha tratado de ajustarse a esta acción, para ello se sugieren las siguientes fases:

1. Análisis del interés y de las necesidades formativas de los estudiantes sobre lo que han recibido o saben sobre dicha asignatura; 2. Definición de una propuesta formativa de acuerdo con el análisis realizado;

3. Planificación de una intervención educativa, con objetivos, contenidos y pautas de acción; 4. Consolidación de esquemas metodológicos de acción en la asignatura, dentro y fuera del aula.

\section{¿Disciplina y/o transversalidad?}

La situación actual en nuestras aulas, debe consistir en establecer una continua comunicación entre las exigencias propias de las diferentes disciplinas clásicas y la problemática del exterior, por lo que se debe intentar ofrecer un tipo de enseñanza que se adecue a la realidad. Desde esta perspectiva, es pertinente aportar respuestas compartidas con otras instituciones sociales del entorno (cuya función educadora está adquiriendo, cada vez, mayor importancia), hasta tal punto que debe ser una idea básica a incorporar a los diseños curriculares. La adaptabilidad de los diseños curriculares a las características idiosincráticas del medio, permite hacer realidad la denominada "transversalidad", o posibilidad de desarrollar objetivos, contenidos y principios de procedimientos a través de permanentes interconexiones entre las distintas áreas, relacionándolas con los contextos sociales dinámicos, que cambian y evolucionan a lo largo del tiempo.

B) Otra interesante experiencia es la Implantación de metodologías activas en asignaturas de últimos cursos por medio de un congreso de alumnos (PLENUM)

Presentada por José María Gómez Hidalgo. Departamento de Sistemas Informáticos de la Escuela Superior Politécnica de la Universidad Europea de Madrid. En II Premios de innovación docente de la Universidad Europea de Madrid.

El PLENUM (congreso), describe una práctica docente desarrollada en la asignatura Procesamiento del Lenguaje Natural (PLN) de quinto curso de la titulación de Ingeniero en Informática, perfil de Inteligencia Artificial, de la Universidad Europea de Madrid. La práctica consiste en la organización y realización de un congreso similar a las conferencias científicas actuales, en todas sus facetas: desarrollo de un trabajo de investigación, redacción de un artículo de investigación, evaluación y revisión del mismo, redacción de la versión final del artículo y presentación ante los otros participantes. En todas estas labores participan los alumnos, y todas ellas contribuyen a la evaluación de su desempeño.

El congreso se denomina PLENUM, y se ha realizado en el curso académico 2003/04 por cuarta vez. Esta experiencia fue puesta en marcha en el año 2000, con el objetivo principal de aumentar la motivación de los alumnos. Con el paso del tiempo, y de manera convergente con el plan de competencias de la UEM y la integración en el Espacio Europeo de Educación Superior, se agregaron los objetivos de integrar distintas metodologías activas de aprendizaje (cooperativo, autónomo y basado en problemas) en una sola experiencia, y de potenciar algunas de las competencias profesionales más demandadas entre los profesionales técnicos: 
1- las habilidades comunicativas;

2- el trabajo en equipo; $y$

3- la capacidad de innovación y creatividad.

A fin de valorar la consecución de estos objetivos, se realizó una evaluación de la iniciativa, tomando como referencia tanto los cuestionarios oficiales UEM de satisfacción del alumnado, como unos cuestionarios específicamente diseñados para ello. Los resultados de la evaluación de esta actividad son muy positivos, alcanzándose importantes incrementos en la satisfacción de los alumnos, y de manera muy especial, en aquellas áreas en las que la iniciativa tenía mayores expectativas de lograr un impacto positivo.

La asignación docente del autor de esta iniciativa implica impartir docencia a los mismos alumnos tanto en cursos intermedios como en los últimos cursos de titulaciones superiores, lo que le ha permitido detectar cambios en la motivación de los alumnos a lo largo de su trayectoria estudiantil, tomando en cuenta las consideraciones siguientes:

1- Dada la cercanía a la obtención del título, los alumnos de últimos cursos pierden el interés por aprender, y lo centran en exclusiva en aprobar. Asimismo, pierden cualquier otro interés que pudieran tener en la mejora de aspectos complementarios de su formación, como los centrados en las competencias profesionales, a pesar del impacto que puedan tener en su futuro profesional.

2- $\mathrm{Al}$ mismo tiempo, los alumnos de últimos cursos se encuentran en un estado óptimo de madurez intelectual, lo que puede permitir sacar máximo partido de su potencial.

El objetivo primordial de nuestra experiencia es aumentar la motivación de los alumnos de últimos cursos, recuperando su interés por aprender, y explotando su madurez intelectual.

Se selecciona la asignatura de Procesamiento del Lenguaje Natural (PLN) por los aspectos que la hacen particularmente adecuada para la implantación de acciones innovadoras de la calidad docente. Estos aspectos, no son en absoluto exclusivos de esta materia, en especial los independientes de los contenidos. Una gran mayoría de las asignaturas optativas de los dos últimos cursos de las titulaciones de informática, comparten estos aspectos, lo que las hace receptáculos idóneos para experiencias similares o paralelas a la propuesta.

Evaluación del PLENUM. La evaluación sistemática de esta iniciativa, orientada a confirmar si se han satisfecho los objetivos planteados en la misma: aumento de la motivación, integración de metodologías de aprendizaje en un todo, y progreso en competencias.

Cualitativamente, opinamos que el PLENUM efectivamente integra elementos de los métodos de aprendizaje citados anteriormente. En referencia al aprendizaje cooperativo, los alumnos trabajan en grupos formales, y existe una dependencia mutua muy estrecha, especialmente en cuanto a la calificación. En cuanto al aprendizaje autónomo, los alumnos disponen constantemente de retroalimentación propia y del profesor, tomado también como referencia los documentos dispuestos por el profesor. Por lo que respecta al aprendizaje basado en problemas (ABP), es destacable que los trabajos propuestos por el profesor integran elementos de distintas partes de la materia teórica, y la obtención de un buen artículo implica la alta comprensión y capacidad de relación de estas partes.

Cuantitativamente, se toman como referencia los cuestionarios de evaluación del profesorado realizados anualmente por la UEM de manera oficial, así como un cuestionario específico planteado por el profesor y orientado concretamente a los aspectos motivacionales y competenciales.

C) El aprendizaje basado en problemas como enfoque pedagógico en la educación en salud. Por Víctor Hugo Dueñas, Bact., M.Sc. Profesor Asociado, Escuela de Bacteriología y Laboratorio Clínico, Facultad de Salud, Universidad del Valle, Cali.

El tutor (docente) debe lograr que el aprendizaje se centre en el estudiante en lugar de centrarlo en el profesor, para ello es vital que tenga un conocimiento amplio y reflexivo del proyecto educativo y del programa de la escuela, debe conocer diversos métodos educacionales o pedagógicos (tutorías, seminarios, uso de problemas, sesiones de grupo, otros) y debe conocer los principios y métodos de evaluación formativa.

En otro sentido, el tutor (docente) debe ser un facilitador del proceso de aprendizaje; debe promover en los estudiantes el pensamiento crítico, la solución de problemas y la toma de decisiones aun en situaciones de incertidumbre; debe estimular que los estudiantes enfoquen los fenómenos desde lo molecular hasta los elementos macrobiológicos, e incluir los aspectos culturales, éticos y socioeconómicos; debe ayudar a los estudiantes a establecer la jerarquía de las hipótesis y de las necesidades de aprendizaje, promover el aprendizaje individual y socializar la información en el sentido de trabajo multidisciplinario; debe coordinar los métodos de evaluación de los estudiantes, asegurar la retroalimentación y la toma de medidas correctivas en el momento adecuado; debe hacer más énfasis en aprender que en enseñar; debe evitar ser visto como figura de autoridad, y fomentar el desarrollo de una atmósfera de confianza y respeto.

El tutor (docente) debe ser un personaje capaz de promover que cada individuo sea sensible a las necesidades y sentimientos colectivos e individuales; debe asegurarse que en los grupos de discusión todos participen, con un ambiente en el que nadie sea un espectador del proceso y debe 
ayudar a los estudiantes a que se atrevan a pensar, a tomar riesgos y a ser capaces de adelantar una hipótesis y luego probar su validez.

Para facilitar y sistematizar las múltiples tareas de los tutores, se pueden diseñar guías que describan las funciones de los tutores, los objetivos mínimos de las propuestas de trabajo y de aprendizaje significativo y los árboles de temas o temáticas propuestos por los diseñadores de los casos o los problemas.

Para no sesgar el trabajo propio de los estudiantes en este enfoque, las guías del tutor no deben ser del conocimiento de los estudiantes. En resumen, las guías del tutor son un recurso que tienen como objetivo dinamizar la tarea de los tutores, brindándoles un norte y un derrotero de lo que el enfoque pedagógico se propone.

Por otra parte, en el trabajo durante el curso se contemplan aquellas actividades que los tutores hacen en la etapa de ofrecimiento del curso que tendrá un enfoque de ABP. Entre esas actividades se cuentan:

1. Presentación del curso o del eje de problemas y explicación del enfoque pedagógico. En la educación tradicional, rara vez se hacen explícitos para los estudiantes los enfoques y los métodos pedagógicos que se utilizarán para favorecer el aprendizaje y la formación. En el enfoque conductista tradicional, los programas de los cursos $\mathrm{o}$ asignaturas se limitan por lo general a incluir una secuencia de contenidos, objetivos y condiciones de evaluación, con poca o ninguna profundización o conceptualización de los mismos.

Cuando se pretende poner en acción un curso o un eje de problemas con enfoque pedagógico de ABP en un currículo que posee un pensum saturado fundamentalmente por el enfoque conductista tradicional, es importante hacer públicas las características del enfoque innovador. Se pretende con la presentación del curso o del eje de problemas, que los estudiantes sean conscientes de la importancia de las áreas de estudio o de las disciplinas que se abordarán $\mathrm{y}$, a su vez con la explicación del enfoque pedagógico se pretende que conozcan y acepten la responsabilidad que exige pasar de un enfoque de aprendizaje pasivo a uno activo, en el que hasta la evaluación formativa depende en gran parte de la objetividad y madurez del estudiante.

2. Dinámica de las discusiones en grupo. Las discusiones en grupo se deben dar en un ambiente que propicie que los estudiantes se sientan en libertad de cuestionar cualquier información que se aporta por parte de ellos mismos o por parte del tutor. Se espera que todos los estudiantes participen en la discusión, bien sea para aportar nuevos datos o para generar nuevos interrogantes producto de la consulta de las fuentes primarias de información, en un ambiente donde el error sea una oportunidad más para aprender.

\section{Asignación de espacios-tiempo para las tutorías}

y la consejería para los estudiantes. La dinámica de trabajo que subyace en el enfoque de ABP le plantea al tutor la necesidad de disponer de espacios-tiempo destinados a la discusión individualizada y a la consejería de los estudiantes. En este enfoque, el estudiante necesitará entrevistarse periódicamente con su tutor para resolver dudas, plantear conflictos y confrontar sus actividades de auto evaluación. Lo anterior significa que para el desarrollo exitoso del enfoque se debe disponer de tiempos relativamente mayores que los destinados o asignados al desarrollo de los enfoques o modelos conductistas.

4. Aplicación de las propuestas de evaluación formativa y evaluación de los medios y recursos. Durante el ofrecimiento del curso, el tutor debe coordinar las actividades de evaluación de los estudiantes y del proceso mismo. Es recomendable evaluar durante el proceso mismo los medios didácticos y los recursos disponibles, con el fin de conocer las impresiones de los estudiantes y las dificultades o facilidades que estos presentan para su aprendizaje. Un ejemplo de este tipo de evaluación es la que se hace de los casos o problemas objeto de estudio como estímulo para el aprendizaje significativo. Las evaluaciones se deben analizar y discutir a medida que ocurren, con el fin de identificar dificultades y proponer medidas correctivas.

Por último, en el trabajo post-curso se incluyen las actividades de análisis de las evaluaciones realizadas durante el ofrecimiento del curso, la toma de decisiones o medidas correctivas que permitan mejorar la propuesta de trabajo para el siguiente grupo de estudiantes y la reflexión sobre las relaciones tutor-estudiantes, tutorconocimiento, estudiantes-estudiantes y estudiantes-conocimiento.

Visto de esta manera, el ABP se puede usar como enfoque para estructurar y desarrollar el currículo de un programa académico o para emplearlo en el desarrollo de algunas asignaturas o cursos como otra estrategia más de trabajo.

$\mathrm{Al}$ aplicar el ABP, las actividades giran en torno a la investigación y discusión de la situación problemática, de este modo, el aprendizaje ocurre como resultado de la experiencia de trabajar en los problemas y la formación se favorece toda vez que es posible reflexionar sobre el modo como se enfrentan los problemas, se proponen las soluciones y sobre las actitudes y aptitudes en torno al enfoque pedagógico que presupone un constante auto-aprendizaje y auto-formación.

En contraste con el modelo de enseñanza conductista tradicional, en el ABP primero se presenta el problema al estudiante, éste elabora un diagnóstico de sus propias necesidades de aprendizaje, busca la información necesaria y regresa de nuevo al problema para analizar y sintetizar la información y plantearse nuevas necesidades de aprendizaje. En este proceso, los estudiantes trabajan de manera cooperativa, 
comparten la información y las experiencias de aprendizaje y tienen la oportunidad de desarrollar habilidades como consecuencia de la observación y reflexión de las situaciones de la vida real.

El objetivo principal en el ABP no es resolver el problema, en su lugar es importante que éste sea visto como un estímulo para la identificar necesidades de aprendizaje y para el desarrollo de habilidades en la búsqueda de la información, su análisis, y el establecimiento de jerarquías en los temas o contenidos de estudio. De este modo, los conocimientos se apropian en relación directa con los problemas o casos y no de manera parcelada y sin relaciones aparentes.

\section{Otras características del enfoque $A B P$, según este} autor:

1- Presupone un proceso motivador en el que los estudiantes son los responsables de su aprendizaje;

2- Es un enfoque que utiliza problemas o casos especialmente diseñados (con una o varias intenciones de estudio) para motivar el aprendizaje de los aspectos más relevantes de la materia o disciplina de estudio;

3- El aprendizaje se centra en el estudiante, no en el profesor o en la transmisión de contenidos agregados;

4- Se trabaja en grupos pequeños de estudiantes. Se asegura que el número ideal de estudiantes es entre 6 y 8 y en todo caso no más de 10;

5- El profesor es un facilitador del proceso, no una autoridad.

Con el enfoque de ABP es factible lograr que el estudiante se responsabilice de su propio proceso de aprendizaje y de formación, desarrolle objetividad para la autoevaluación; desarrolle habilidades para la comunicación, las relaciones interpersonales y el trabajo en equipo; adquiera el hábito del auto-aprendizaje como un mecanismo que garantice su aprendizaje fuera de la escuela, es decir, el auto-aprendizaje y la auto-formación como acto cotidiano de por vida.

El enfoque de $\mathrm{ABP}$ favorece los cuatro aprendizajes fundamentales para el cumplimiento de las misiones propias de la educación superior:

$\leftarrow$ En primer lugar, favorece el aprender a conocer o a aprender, enfocando esos aprendizajes más al dominio de los instrumentos propios del saber, que a la adquisición de conocimientos clasificados y codificados de manera arbitraria por los diseñadores de la estructura curricular.

En el proceso de aprender a aprender, se debe favorecer el ejercicio de la atención, la memoria asociativa, y el pensamiento crítico. Para asegurar la competencia y la calidad en la práctica profesional, la universidad a través de los procesos formativos debe proponer actividades que además de estimular la ejercitación repetida, desarrollen los procesos de pensamiento crítico. El proceso de pensamiento crítico se puede ilustrar como una habilidad adquirible que demanda competencias para evaluar, intuir, debatir, sustentar, opinar, decidir y discutir entre otras. Estas competencias se pueden desarrollar, por lo menos en parte, en los espacios tiempo destinados a la socialización del conocimiento que se ofrecen en el enfoque de ABP. Ahora bien, aunque las competencias mencionadas no constituyen el pensamiento en sí mismo, son importantes para el desarrollo del pensamiento intuitivo y problematizador, sólo si se consideran y observan en un contexto de rigor académico, es decir, no con una acepción superficial del concepto de pensar.

$\leftarrow$ En segundo lugar, el enfoque favorece el aprender a hacer desde la perspectiva de encontrar la mejor manera de poner en práctica los conocimientos para transformar el entorno, adaptándose al mercado de trabajo y sin menospreciar la instrucción rigurosa que permita la realización de tareas bien definidas propias del área de conocimiento de la profesión.

$\leftarrow$ En tercer lugar, se favorece con este enfoque el aprender a convivir, toda vez que en los espacios de socialización del conocimiento, el estudiante aprende a comunicarse, a trabajar con los demás y a solucionar conflictos que ocurren como producto de las interrelaciones en los grupos tutoriales.

En esa dinámica de trabajo los estudiantes y los tutores gradualmente se reconocen a sí mismos, reconocen sus potencialidades y sus debilidades y se proponen objetivos individuales y comunes. El conocimiento de sí mismo y el establecimiento en los grupos de objetivos comunes son condiciones forzosas para el conocimiento del otro.

Finalmente, el enfoque favorece el aprender a ser, al permitir que los estudiantes y los tutores expongan sus personalidades y al fomentar la autonomía de juicio, la responsabilidad personal y social dentro de un ambiente de comunicación que asevera ser de doble vía, de pluralismo y de respeto por las diferencias.

Con el propósito de que el debate quede abierto, es conveniente decir que el enfoque de $\mathrm{ABP}$ no es el único que se puede abordar para el diseño de los currículos o asignaturas en las universidades. Con seguridad lo ideal será una mezcla de varios enfoques pedagógicos al diseño de currículos orientados por procesos y no por objetivos. En este sentido, el autor propone y retoma siete principios pedagógicos que se pueden introducir en los currículos orientados por procesos:

1. Iniciar y desarrollar en los planes de las asignaturas un proceso de planteamiento de preguntas (método de indagación), esto contribuiría al desarrollo del pensamiento (lógica) en el estudiante y en el profesor.

2. Incorporar metodologías de investigación donde 
los estudiantes puedan buscar datos e informes para responder a las preguntas que se han planteado y aplicarlos a nuevas áreas.

3. Construir un ambiente propicio para desarrollar en el estudiante la capacidad de utilizar diversas fuentes de información de primera mano, como datos a partir de los cuales pueda desarrollar hipótesis y extraer conclusiones.

4. Brindar espacios de discusiones en clase, en las que los estudiantes aprendan tanto a escuchar a los demás, como a exponer sus propios puntos de vista en procura de mejorar sus habilidades de comunicación (retórica).

5. Legitimar la búsqueda, es decir, aprobar y apoyar discusiones abiertas en las que no se hallan respuestas definitivas a multitud de cuestiones, esto contribuiría al desarrollo de la capacidad de tomar decisiones aun en situaciones de incertidumbre.

6. Animar a los estudiantes a reflexionar respecto de sus propias experiencias y a compartirlas en procura de la socialización del conocimiento.

7. Otorgar un nuevo papel al profesor para que se convierta en un recurso más que en una autoridad, es decir se debe brindar espacios para que el profesor se acerque al papel de tutor o guía del estudiante en su proceso de aprendizaje y de formación.

\section{Fuentes de Información Consultadas}

1. Crespo, Juan Carlos. Diálogo Global 13 de diciembre del 2005 4:00 a 6:40 p.m. (hora de Washington D.C.) , 4:00 a 6:40 p.m. (hora de Lima) en Videoconferencias Infocentros El Salvador. Director del Centro para el Magisterio Universitario de la Pontificia Universidad Católica del Perú (MAGIS-PUCP) GDL PERÚ PUCP.jcrespo@pucp.edu.pe

2. Gómez Hidalgo, José María (2005). Implantación de metodologias activas en asignaturas de últimos cursos por medio de un congreso de alumnos (PLENUM),
Departamento de Sistemas Informáticos de la Escuela Superior Politécnica de la Universidad Europea de Madrid. Memoria descriptiva. II Premios de innovación docente de la Universidad Europea de Madrid, 29 de noviembre de 2005.jmgomez@uem.es ; consultado el 09/06/06, disponible en:

http://www.esp.uem.es/ jmgomez/

3. Limón Domínguez, Dolores. Universidad de Sevilla. Criterios de selección de contenidos curriculares a partir de un programa de análisis de necesidades. Presentado en XVIII Seminario Interuniversitario de Teoría de la Educación "La educación obligatoria. Competencias Básicas del Alumno". Universidad de Extremadura, noviembre de 1999. En línea, consultado el 09/06/06

4. Pedraz Marcos, Azucena, Antón Nardiz, María Victoria; García González; Asunta. Revista de la Red Estatal de Docencia Universitaria vol $3 N^{\circ} 2$, Artículo en pdf: Observación de una tutoría de aprendizaje basado en problemas $(A B P)$ dentro de la asignatura "Legislación y ética profesional en enfermeria" ( $p p$ 8793). Profesoras de la Escuela Universitaria de Enfermería de la Comunidad de Madrid, (en línea, consultado el 19/06/06), disponible en:

Http://www.uc3m/diceimbre2003/activospdf 5. Red Panamericana para el Aprendizaje Basado en Problemas (PAN-ABP) o Pan-American Network or Problem-Based Learning (PAN-PBL). EL APRENDIZAJE BASADO EN PROBLEMAS: ¿REQUIERE TRANSFORMACIÓN CURRICULAR O ES POSIBLE UN CURRÍCULO DISCIPLI NAR? 27 de octubre del 2005, disponible en: http://www.udel.edu/pan-pbl/

6. Víctor Hugo Dueñas, Bact., M.Sc. El aprendizaje basado en problemas como enfoque pedagógico en la educación en salud. Profesor Asociado, Escuela de Bacteriologia y Laboratorio Clínico, Facultad de Salud, Universidad del Valle, Cali. (En línea) consultado el 9/06/2006 disponible en

http://64.233.187.104/search?q=cache:CiJ6mcqmW1 gJ:colombiamedica.univalle.edu.co/VOL32NO4/apre ndizaje.pdf 